\title{
Treatment of severe periodontic-endodontic combined lesions with minocycline hydrochloride ointment combined with mineral trioxide aggregate
}

\author{
WEN SHAO ${ }^{1 *}$, FENG XIAO $^{2 *}$, ZHONG-XIN XU $^{3}$, RUI-HAN REN ${ }^{4}$, YONG WANG $^{5}$ and YI-QUN WU ${ }^{1}$ \\ ${ }^{1}$ The 2nd Dental Center, Ninth People's Hospital, Shanghai Jiao Tong University School of \\ Medicine, Shanghai Key Laboratory of Stomatology, Shanghai 200011; ${ }^{2}$ Department of Dentistry, \\ The Second Affiliated Hospital of Anhui Medical University, Hefei, Anhui 230600; ${ }^{3}$ Department of \\ Counterterrorism Research, Zhejiang Police College, Hangzhou, Zhejiang $310053 ;{ }^{4}$ Department of Dentistry, \\ People's Hospital of Linyi, Linyi, Shandong 276000; ${ }^{5}$ Department of Oral and Maxillofacial Surgery, \\ The Ninth People's Hospital, College of Stomatology, School of Medicine, Shanghai Jiao Tong University, \\ Shanghai Key Laboratory of Stomatology, Shanghai 200011, P.R. China
}

Received September 21, 2017; Accepted January 10, 2018

DOI: $10.3892 / \mathrm{etm} .2018 .6341$

\begin{abstract}
Periodontic-endodontic combined lesions affect the quality of life of patients. The present study aimed to explore a way to achieve maximal effect in treating periodontic-endodontic combined lesions by using a combination of minocycline hydrochloride ointment (MHO) and mineral trioxide aggregate (MTA). In the present study, 294 patients were randomly divided into four groups: Control group, MHO group, MTA group and combination group. Endodontic and periodontic therapy was applied simultaneously on the anterior and posterior teeth of patients, and patient characteristics, gingival index (GI) and probing depth (PD) were noted when the patients were treated with the different strategies. Reexamination was performed 4 weeks after treatment and follow-up was performed 2 years later. The present study demonstrated that medical history, root filling and periapical condition within the patients were deemed as key factors in determining the success of treatment of periodontic-endodontic
\end{abstract}

Correspondence to: Professor Rui-Han Ren, Department of Dentistry, People's Hospital of Linyi, 27 East Jiefang Road, Linyi, Shandong 276000, P.R. China

E-mail: 362233066@qq.com

Dr Yong Wang, Department of Oral and Maxillofacial Surgery, The Ninth People's Hospital, College of Stomatology, School of Medicine, Shanghai Jiao Tong University, Shanghai Key Laboratory of Stomatology, 639 Zhizaoju Road, Shanghai 200011, P.R. China E-mail: wangyong1405@126.com

*Contributed equally

Key words: periodontics, endodontics, minocycline hydrochloride ointment, mineral trioxide aggregate, gingival index, periodontal depth combined lesions. The effect of the combination treatment significantly exceeded that of either treatment alone $(\mathrm{P}<0.05)$. In addition, the teeth were significantly improved for patients with pathological types I, II and III in the combination group compared with either treatment group alone $(\mathrm{P}<0.05)$. Furthermore, compared with the MTA and MHO groups alone, the combination group demonstrated a significant decrease in PD and GI in severe periodontic-endodontic combined lesions. In conclusion, the present findings implied that combination treatment is significantly superior to single treatments applied to patients with severe periodontic-endodontic combined lesions.

\section{Introduction}

Periodontic-endodontic combined lesions are diseases of dental pulp tissue, regularly caused by bacterial infection from severe dental caries, periodontitis, tooth fracture or traumatic injuries $(1,2)$. Infected dental pulp may lead to severe pain or clinical symptoms, such as abscess. It has the possibility to turn lethal, thus requires cautious and thorough treatment (2). The control of plaque is an early stage periodontal treatment method (3). The strict control of plaque is a necessary measure for periodontal treatment as excessive plaque may limit the effectiveness of other treatments. Therefore plaque control is important in the treatment of periodontal disease and is managed throughout the whole process (3). Patients with periodontic-endodontic combined lesions are treated with pulp therapy without periodontal treatment when the cases have typical pulp symptoms, such as a sudden, intense pain in the mouth (4). If the underlying cause is not treated, although clinical symptoms may improve following dental pulp treatment, periodontal disease may continue to develop, consequentially lowering the effective rate of treatment (4).

Mineral trioxide aggregate (MTA) (4) has attracted attention since it was first reported by Lee et al (5) in 1993. 
Certification for its clinical use was granted by the Food and Drug Administration in 1993 and it was adopted in dental therapy in following years (6). MTA has low cytotoxicity, agreeable biocompatibility and has been certified in vivo and in vitro $(7,8)$. When MTA is used as a repair material in the apical zone, it has been observed to induce almost no inflammatory reaction, and almost all samples with MTA surfaces demonstrate new cementum formation (8). It has been demonstrated that when MTA is applied as a repair material in a treatment group, cementum was observed in the perforation area with almost no inflammation; however, necrosis of periodontal tissues was observed in the control groups that were treated with other materials (9). In addition, almost all the periodontal tissues in the treatment group were restored in 6 months, and cellular cementum had formed between cementum and alveolar bone, accompanied by the formation of periodontal ligaments (9). MTA has been applied directly for pulp capping and pulp cutting, which has been indicated to induce almost no inflammatory reaction and promoted the development of the root (10). MTA has received much attention due to its favorable tissue compatibility, high bioactivity and low cytotoxicity (11). Additionally, MTA effectively reduces the production of micro leakage in dental diseases, increases the success rate of root canal therapy, promotes the proliferation of dental pulp stem cells to preserve the vitality of the pulp and also improves the success rate of root tip induction $(11,12)$. However, the application of MTA has shortcomings, including a slow cure rate and its application requires sterile conditions, which limits its application to the clinic.

In the affected tooth, the mixed infection of anaerobic bacteria predominantly exists in the periodontal pocket and pulp, both of which affect and spread the infection and disease to each other, which increases the difficulty of clinical treatment (13). Minocycline is the main constituent of minocycline hydrochloride ointment (MHO), which has a wide antibacterial spectrum, strong antibacterial activity, high efficiency and long lasting effects (14). Furthermore, its antibacterial effect is three times stronger than that of the single tetracycline antibiotic $(14,15)$. As a kind of local periodontal sustained release drug, MHO becomes a hard layer of membrane when it encounters water, allowing it to slowly release the drug into the periodontal pocket. The high local concentration of MHO significantly relieves clinical periodontal symptoms $(16,17)$. However, MHO may induce dysbacteriosis, which may lead to vitamin deficiency. Severe vitamin deficiency cases may result in liver damage, kidney damage and other adverse reactions, which require replacement of the treatment type or a combination therapy that ameliorates the outcome (16).

Periodontic-endodontic combined lesions are fairly common clinically, and the periodontal tissue and dental pulp tissue communicate with each other anatomically, making treatment difficult. MHO is a highly efficient, long-lasting, strong broad-spectrum antimicrobial with sustained-release preparations, and MTA has high histocompatibility, edge sealing, high biological activity and low cytotoxicity. However, the side effects of MHO cannot be dismissed. Thus, the present research aimed to determine the effectiveness and feasibility of MHO and MTA combination treatment in severe periodontic-endodontic combined lesions.

\section{Patients and methods}

Patients. Ethical approval was granted by the Medical Ethics Committee of Shanghai Jiaotong University (Shanghai, China; reference no. JTRT2010051531). Informed signed consent was gathered from the participants prior to initiation of the study. Patients with periodontic-endodontic combined lesions were selected from the Department of Stomatology, Shanghai Ninth People's Hospital (Shanghai, China) between August 2010 and July 2014. The diagnosis was performed by two independent researchers. Inclusion criteria were as follows: i) Periodontitis: $3-10 \mathrm{~mm}$ of periodontal pocket depth, $1-3^{\circ}$ of tooth mobility (16), $1-3^{\circ}$ of root furcation and loss of lamina as shown in X-ray examination; and ii) pulpitis and periapical periodontitis: Continuous dental malaise, dull or negative in test for pulp vitality and periapical sparse area as shown in X-ray examination. Exclusion criteria were as follows: i) Patients with deciduous teeth; ii) patients unable to take root canal therapy; iii) patients unable to take timely reexamination; iv) according to two researchers with clinical experience, patients with systemic diseases that affect the treatment effect, including severe kidney disease, diabetes, hyperthyroidism and acquired immune deficiency syndrome (18); and v) patients that had received other therapies in last 3 months.

A total of 212 patients were selected for the present study. There were 92 males and 116 females, with an age range of 20-80 years. A total of 294 teeth were included in the present experiment: 168 anterior teeth and 126 posterior teeth. The patients were randomly divided into four groups: Control group (56 cases and 75 teeth), MTA group (52 cases and 73 teeth), MHO group (52 cases and 71 teeth) and combination group (52 cases and 75 teeth). There were three types of etiology according to the cause of the disease: Type I, periodontal lesions caused by endodontic diseases; type II, endodontic lesions caused by periodontal diseases; and type III, coexistence of two lesions. The pathological changes of pulp were classified into four grades according to the standard reported by Mazur and Massler (18), including grade I, grade II, grade III and grade IV. The teeth were divided into four levels according to the pulp pathological grade: Level I, II, III and IV (18). These levels and grades indicate increasingly severe pathological damage.

Experimental materials. 2\% MHO (trade name, Perioline) was purchased from Sunstar, Inc., (Osaka, Japan). MTA was purchased from Dentsply Sirona (York, PA, USA).

Specimen preparation and hematoxylin and eosin staining. For patients who decided to remove affected teeth regardless of severity, the extraction was performed following additional signed consent by the patient. Subsequent to tooth extraction, the teeth were fixed in $10 \%$ neutral formalin for 3 days and then washed using water flushing. Following this, the relative two axial surfaces of the teeth were evenly ground along the tooth body using a high-speed turbine with diamond sand until the complete morphology of the marrow cavity was clearly visible through the hard tissue of the tooth. The teeth were fixed in $10 \%$ neutral formalin at room temperature for 1 week to ensure that the pulp was fully fixed. The teeth were 
removed when they were soft enough to perforate with an expanding needle, and then washed with flowing water. The tooth slices were removed from the $10 \%$ formaldehyde solution and douched with $0.85 \%$ saline. The liquid on the surface was wiped away carefully with filter paper. Subsequently, the samples were dehydrated with $95-100 \%$ alcohol in an automatic hydroextractor overnight. A pathological tissue embedding machine (cat no. 8330; Thermo Fisher Scientific, Inc., Waltham, MA, UAS) was utilized to slice the paraffin embedded tooth tissue into slices of $3 \mu \mathrm{m}$. The slices were tweezed onto a glass slide gently and the folds were stretched out. The ready-made slice was dewaxed twice using xylene, for $10 \mathrm{~min}$ each, and then douched in saline. The resultant slice was stained for $5 \mathrm{~min}$ at room temperature using hematoxylin. The glass slide was douched and then $0.5 \%$ eosin was used for staining for $30 \mathrm{sec}$ at room temperature. The slice was dehydrated with alcohol again and dealcoholized with xylene. Neutral gum (cat. no. BD5044; Bioworld Technology, Inc., St. Louis Park, MN, USA) was dropped on the slice so that the cover slip was replaced. The resultant slice was observed under a light microscope at magnification x200 and images were captured.

Treatment method. Local treatment on the lesion, including rinsing and disinfection, was performed prior to the treatment method of the present study. At the same time, $500 \mathrm{mg}$ broad-spectrum antimicrobial agents Amoxicillin Capsules (cat. no. A662203; Sangon Biotech Co., Ltd., Shanghai, China) and $400 \mathrm{mg}$ metronidazole (cat. no. A600633; Sangon Biotech Co., Ltd.) was taken orally, three times a day for 1 week prior to the present study. Root canal preparation and root canal disinfection were performed in the four groups. Routine root canal therapy was performed in the four study groups. Following disinfection, a drug conveyor was inserted into the front end of the root tip $\sim 1 / 5$ deep, which injected the tip of the tooth with the desired drug. When the MTA paste [Zinc oxide glycerin paste (Desitin Pharma Ltd., Milton Keynes, UK) for the control group] was overflown from the root canal orifice, injection and simultaneous extraction of the conveyer was continued gently. Excess paste was removed using a cotton pad. Zinc oxide glycerin paste and apical root canal was used for filling in the control group. All procedures were performed by the same senior specialist.

Evaluation of curative effect standard. Clinical effects were collected and evaluated in patients following treatment, according to associated literature $(18,19)$, as follows: Cured, periodontal pockets are shallower, no conspicuous exsertion was noticed in the bifurcation area, no loosening in percussion, good masticatory function, no shallow areas as shown in X-ray examination and the formation of new lamina dura; effective, periodontal pockets shallowed but not conspicuous, $1^{\circ}$ exsertion in bifurcation area, $1^{\circ}$ loosening in percussion, unable to chew hard food, shrunken shallow areas as demonstrated in an X-ray and partial formation of lamina dura; and ineffective, depth of periodontal pockets not changed, positive results in percussion, poor masticatory function, and same or enlarged shallow area in periapical and periodontal area. The proportion of cured and effective teeth was recorded as the total effective rate.
Probing depth (PD) and gingival index (GI) were recorded as clinical indices for periodontal condition (18). Evaluation of therapeutic effect was as follows: Significantly effective, PD declining $>2 \mathrm{~mm}$, GI decreasing to $1 / 2$, no clinical symptoms and a reduced degree of loosening; effective, PD declining $>1 \mathrm{~mm}$, GI decreasing to $1 / 3-1 / 2$, no improvement in the degree of loosening and no clinical symptoms; and ineffective, no improvement in clinical symptoms, no improvement in PD or GI and no improvement in degree of loosening.

Follow-up. The patients were followed up every quarter of a year for 2 years after treatment and statistically analyzed by their conditions. Evaluation standard was performed as aforementioned.

Statistical analysis. SPSS (version 21; IBM Corp., Armonk, NY, USA) was used for data analysis. Measurement data were presented as the mean \pm standard deviation. Analysis of variance followed by the Dunnett's post hoc multiple comparisons test were applied to analyze multiple groups. The $\chi^{2}$ test was used for comparison between the groups. Factors affecting the root filling effect, including age, sex, tooth position, etiology, medical history, root canal filling, root filling method and periapical situation, were analyzed by logistic regression analysis. $\mathrm{P}<0.05$ was considered to indicate a statistically significant difference.

\section{Results}

Pathological changes of chronic periodontic-endodontic combined lesions. The pathological changes of the dental pulp tissue were observed under a microscope, the typical images of each grade are demonstrated in Fig. 1. The data indicated that the pathological changes of dental pulp in patients with mild periodontitis were the highest in grade I and II, grade III was slightly less and grade IV was the lowest. In addition, the severe periodontitis group suffering from the most notable dental pathological changes (including periodontal cysts) were present in level IV, level I demonstrated the least pathological changes, and grade II and III demonstrated slightly more changes than grade I. Representative images was taken at magnification $\mathrm{x} 200$. The number of periodontal cysts increased from Grade I to IV. In Fig. 2, X-ray images captured prior to treatment of patients' teeth were used for classification according to their pathological damage. The white arrows indicate lesions of different grades; the lesions become larger and occur in larger numbers at higher grades. These data implied that mild periodontitis may be associated with mild dental pulp stimulation while severe periodontitis may be associated with stronger stimulation. Of note, the staining of all samples in Fig. 1 was the same. The inhomogeneous staining of the grade IV sample may be due to the restore condition of the slide, which may contaminate the tissue color. This does not affect the outcome.

Medical history, root filling and periapical condition are critical factors in treatment of periodontic-endodontic combined lesions. In the present study, patients with severe periodontic-endodontic combined lesions were the main focus and related factors were further studied. Factors affecting the 

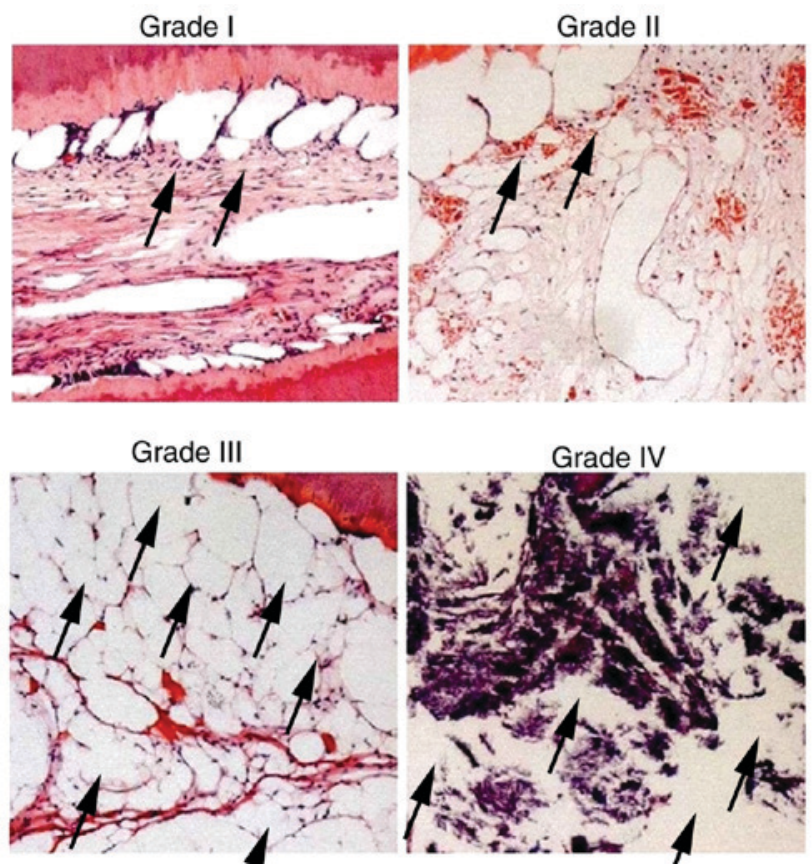

Figure 1. Classification of pathological changes in patients with different severities of periodontic-endodontic combined lesions. The number and severity of the periodontal cysts increased from Grade I to IV. Representative images were captured at magnification of $\times 200$. Black arrows indicate pathological changes.

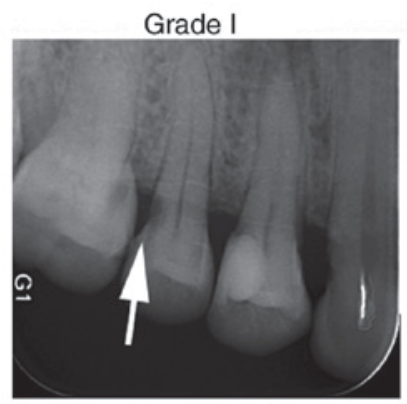

Grade III

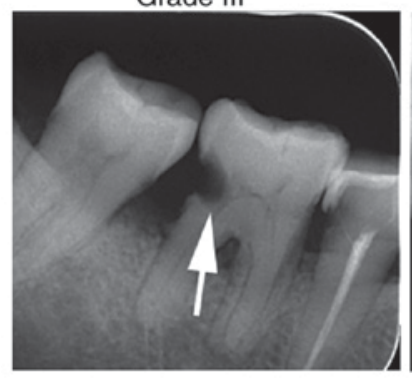

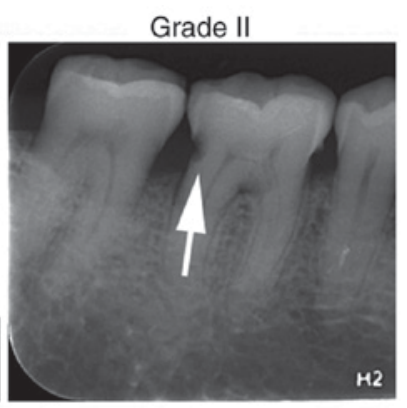

Grade IV

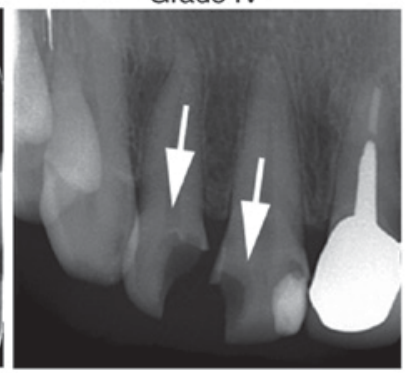

Figure 2. X-rays captured prior to treatment of patients' teeth were used to classify the patients according to the pathological damage. White arrows indicate lesions of different grades. The lesions become larger and increase in number with each grade.

root filling effect, including age, sex, tooth position, etiology, medical history, root canal filling, root filling method and periapical situation, were analyzed by logistic regression analysis. The results demonstrated that medical history $(\mathrm{P}=0.023)$, periapical situation $(\mathrm{P}=0.009)$ and root canal filling $(\mathrm{P}=0.347)$ were closely related to clinical efficacy. Other factors, including age, sex, tooth position, etiological factor and root
Table I. Factors relevant to patients with severe periodonticendodontic combined lesions analyzed by logistic regression.

\begin{tabular}{lcrc}
\hline Factor & $\begin{array}{c}\text { B regression } \\
\text { coefficient }\end{array}$ & \multicolumn{1}{c}{$X^{2}$} & P-value \\
\hline Age & 0.082 & 0.285 & 0.781 \\
Sex & 0.352 & 1.529 & 0.263 \\
Tooth position & 0.423 & 0.011 & 0.118 \\
Etiology & 0.769 & 0.075 & 0.904 \\
Medical history & 1.966 & 9.832 & 0.023 \\
Medical expense & 3.350 & 18.923 & 0.012 \\
Root canal & 0.967 & 1.781 & 0.347 \\
filling measure & & & \\
Periapical & 1.542 & 23.109 & 0.009 \\
condition & & & \\
\hline
\end{tabular}

filling method, were not observed to be significantly associated with clinical efficacy (Table I). These results suggest that these indexes affect the joint lesion of periodontal pulp, which may enable further improvement of the treatment effect.

Efficacy of combination therapy for anterior and posterior teeth is greater than that of MTA or MHO therapy alone in patients with severe periodontic-endodontic combined lesions. In order to study the curative effect of combination therapy in patients with severe periodontic-endodontic lesions, the various therapy strategies were used to treat the patients and statistical analysis was performed to compare the effects in the different groups. The results demonstrated that the curative effect for the anterior and posterior teeth did not differ significantly in patients in the control group and three experimental groups $(\mathrm{P}>0.05)$. Subsequently, the curative effect between the different experimental groups was analyzed. The data indicated that the curative effect for the anterior and posterior teeth of the patients in the experimental groups was significantly increased compared with the effects observed in the control group $(\mathrm{P}<0.05$; Table II). The present data also revealed that the curative effect for the anterior and posterior teeth of the patients in the MTA group and MHO group was not statistically significant $(\mathrm{P}>0.05)$. However, the curative effect for the anterior and posterior teeth of the patients in the combined treatment group was significantly increased compared with the effects observed in the MTA and MHO groups $(\mathrm{P}<0.05$; Table II). These data suggest that there was no significant difference between the effects on the anterior and posterior teeth of the patients when the patients were treated with MTA or MHO alone. However, the curative effect for the anterior and posterior teeth of the patients was significantly improved when the patients received the combined treatment, indicating that the combination therapy had a superior curative effect for the anterior and posterior teeth of the patients with severe periodontic-endodontic lesions.

Combination treatment is more effective in patients with pathological types I, II and III severe periodontic-endodontic combined lesions. In order to study the curative effect on 
Table II. Comparison of clinical effects of treatment on anterior and posterior teeth in patients with severe periodontic-endodontic combined lesions.

\begin{tabular}{|c|c|c|c|c|c|c|}
\hline \multirow[b]{2}{*}{ Group } & \multirow[b]{2}{*}{ Tooth } & \multirow[b]{2}{*}{$\mathrm{n}$} & \multicolumn{3}{|c|}{ Treatment effect, n (\%) } & \multirow[b]{2}{*}{ Total effective rate, $\mathrm{n}(\%)$} \\
\hline & & & Cured & Effective & Ineffective & \\
\hline \multirow[t]{2}{*}{ Control } & Anterior & 40 & $13(32.5)$ & $8(20)$ & $19(47.5)$ & $21(52.5)$ \\
\hline & Posterior & 35 & $6(31.6)$ & $9(25.7)$ & $20(57.1)$ & $15(42.9)$ \\
\hline \multirow[t]{2}{*}{ MTA } & Anterior & 38 & $17(44.7)$ & $11(28.9)$ & $10(26.3)$ & $28(73.7)^{\mathrm{a}}$ \\
\hline & Posterior & 35 & $14(40.0)$ & $7(20.0)$ & $14(40.0)$ & $21(60.0)^{\mathrm{b}}$ \\
\hline \multirow[t]{2}{*}{ MHO } & Anterior & 37 & $19(52.4)$ & $8(21.6)$ & $10(27.0)$ & $27(73.0)^{\mathrm{a}}$ \\
\hline & Posterior & 34 & $12(35.3)$ & $10(29.4)$ & $12(35.3)$ & $22(64.7)^{b}$ \\
\hline \multirow[t]{2}{*}{ Combination } & Anterior & 39 & $25(64.1)$ & $12(30.8)$ & $2(12.8)$ & $37(94.9)^{\mathrm{a}, \mathrm{c}, \mathrm{e}}$ \\
\hline & Posterior & 36 & $20(55.6)$ & $13(36.1)$ & $3(8.3)$ & $33(91.7)^{\mathrm{b}, \mathrm{d}, \mathrm{f}}$ \\
\hline
\end{tabular}

${ }^{\text {aP }}<0.05$ vs. anterior tooth in control group; ${ }^{b} \mathrm{P}<0.05$ vs. posterior tooth in control group; ${ }^{\mathrm{C}} \mathrm{P}<0.05$ vs. anterior tooth in $\mathrm{MTA}$ group; ${ }^{\mathrm{d}} \mathrm{P}<0.05$ vs. posterior tooth in MTA group; ${ }^{\mathrm{e}} \mathrm{P}<0.05$ vs. anterior tooth in $\mathrm{MHO}$ group; ${ }^{\mathrm{f}} \mathrm{P}<0.05$ vs. posterior tooth in $\mathrm{MHO}$ group. MTA, mineral trioxide aggregate; MHO, minocycline hydrochloride ointment.

Table III. Clinical effects of treatments in patients with different pathological types of periodontic-endodontic combined lesions.

\begin{tabular}{|c|c|c|c|c|c|c|}
\hline \multirow[b]{2}{*}{ Group } & \multirow[b]{2}{*}{ Pathological type } & \multirow[b]{2}{*}{$\mathrm{n}$} & \multicolumn{3}{|c|}{ Treatment effect, n (\%) } & \multirow[b]{2}{*}{ Total effective rate, $\mathrm{n}(\%)$} \\
\hline & & & Cured & Effective & Ineffective & \\
\hline \multirow[t]{3}{*}{ Control } & I & 28 & $9(32.1)$ & $5(17.9)$ & $14(50.0)$ & $14(50.0)$ \\
\hline & II & 30 & $7(23.3)$ & $6(20.0)$ & $17(56.7)$ & $13(43.3)$ \\
\hline & III & 17 & $7(41.2)$ & $2(11.8)$ & $8(47.1)$ & $9(52.9)$ \\
\hline \multirow[t]{3}{*}{ MTA } & I & 27 & $11(40.7)$ & $5(18.5)$ & $11(40.7)$ & $16(59.2)^{\mathrm{a}}$ \\
\hline & II & 29 & $12(41.4)$ & $8(27.6)$ & $9(31.0)$ & $20(69.0)^{\mathrm{b}}$ \\
\hline & III & 17 & $8(47.1)$ & $4(23.5)$ & $5(29.4)$ & $13(70.6)^{c}$ \\
\hline \multirow[t]{3}{*}{ MHO } & I & 27 & $13(48.1)$ & $5(18.5)$ & $9(33.3)$ & $18(66.7)^{\mathrm{a}}$ \\
\hline & II & 26 & $9(34.6)$ & $8(30.8)$ & $9(34.6)$ & $17(65.4)^{\mathrm{b}}$ \\
\hline & III & 18 & $8(44.4)$ & $6(33.3)$ & $4(22.2)$ & $14(77.8)^{\mathrm{c}}$ \\
\hline \multirow[t]{3}{*}{ Combination } & I & 29 & $23(79.3)$ & $4(13.8)$ & $2(6.9)$ & $27(93.1)^{\mathrm{a}, \mathrm{d}, \mathrm{g}}$ \\
\hline & II & 30 & $22(73.3)$ & $6(20.0)$ & $2(6.7)$ & $28(93.3)^{\mathrm{b}, \mathrm{e}, \mathrm{h}}$ \\
\hline & III & 16 & $12(75.0)$ & $3(18.8)$ & $1(6.3)$ & $15(93.8)^{\mathrm{c}, \mathrm{f}, \mathrm{i}}$ \\
\hline
\end{tabular}

${ }^{\mathrm{a}} \mathrm{P}<0.05$ vs. type I in control group; ${ }^{\mathrm{b}} \mathrm{P}<0.05$ vs. type II in control group; ${ }^{\mathrm{C}}<0.05$ vs. type III in control group; ${ }^{\mathrm{d}} \mathrm{P}<0.05$ vs. type I in $\mathrm{MTA}$ group; ${ }^{\mathrm{e}} \mathrm{P}<0.05$ vs. type II in MTA group; ${ }^{\mathrm{P}}<0.05$ vs. type III in MTA group; ${ }^{9} \mathrm{P}<0.05$ vs. type I in $\mathrm{MHO}$ group; ${ }^{\text {}} \mathrm{P}<0.05$ vs. type II in $\mathrm{MHO}$ group; ${ }^{\mathrm{P}}<0.05$ vs. type III in MHO group. MTA, mineral trioxide aggregate; $\mathrm{MHO}$, minocycline hydrochloride ointment.

patients with severe periodontic-endodontic combined lesions, the curative effect on various disease types following treatment with different strategies was analyzed. The results revealed that the curative effects for the type I, II and III periodontal pulp joint lesions of the patients in the control group or experimental groups were not statistically significant $(\mathrm{P}>0.05)$. Subsequently, the curative effect between the different experimental groups was analyzed. The data revealed that the curative effect for the type I, II and III periodontal pulp joint lesions of the patients in the experimental groups was significantly increased compared with the type I, II and III periodontal pulp joint lesions in the control group, respectively $(\mathrm{P}<0.05$; Table III). The present data also indicated that the curative effect for the type I, II and III periodontal pulp joint lesions of the patients in the
MTA group and MHO group was not statistically significant ( $>0$ 0.05). However, the curative effects for the type I, II and III periodontal pulp joint lesions of the patients in the combined treatment group were significantly increased compared with those in the MTA and MHO groups, respectively $(\mathrm{P}<0.05$; Table III). These data imply that there was rarely a significant difference for the type I, II and III periodontal pulp joint lesions of the patients when the patients were treated with MTA or MHO alone; however, the curative effect for the type I, II and III periodontal pulp joint lesions of the patients was significantly improved when the patients received combined treatment. This suggests that the combination therapy had a superior curative effect for the different types of periodontal pulp joint lesions of the patients with severe periodontic-endodontic lesions. 
Table IV. Clinical effect in 2-year follow-up.

\begin{tabular}{|c|c|c|c|c|c|}
\hline \multirow[b]{2}{*}{ Group } & \multirow[b]{2}{*}{$\mathrm{n}$} & \multicolumn{3}{|c|}{ Treatment effect, n (\%) } & \multirow[b]{2}{*}{ Total effective rate, $\mathrm{n}(\%)$} \\
\hline & & Cured & Effective & Ineffective & \\
\hline Control & 56 & $13(23.2)$ & 11 (19.6) & $32(57.1)$ & $24(42.9)$ \\
\hline MTA & 52 & $21(39.6)$ & $10(18.9)$ & $21(40.4)$ & $31(58.5)^{\mathrm{a}}$ \\
\hline MHO & 52 & $20(36.4)$ & $14(25.5)$ & $18(34.6)$ & $34(60.7)^{\mathrm{a}}$ \\
\hline Combination & 52 & $33(57.9)$ & $14(26.9)$ & $5(9.6)$ & $47(90.3)^{\mathrm{a}-\mathrm{c}}$ \\
\hline
\end{tabular}

${ }^{\text {a }} \mathrm{P}<0.05$ vs. control group; ${ }^{b} \mathrm{P}<0.05$ vs. MTA group; ${ }^{\mathrm{c}} \mathrm{P}<0.05$ vs. MHO group. MTA, mineral trioxide aggregate; MHO, minocycline hydrochloride ointment.

Combination treatment decreases the PD and GI index in patients with severe periodontic-endodontic combined lesions. In order to explore the efficacy of treatment strategies for patients with severe periodontic-endodontic combined lesions, GI and PD were further statistically analyzed prior to and following treatment in patients. As demonstrated in Fig. 3, there was no significant difference between the experimental groups and the control group prior to treatment $(\mathrm{P}>0.05)$, which implied that the experimental results were comparable. In addition, the data revealed that the GI was decreased significantly in each treatment group compared with that in the control group after 4 weeks of treatment $(\mathrm{P}<0.05)$. PD was not significantly different in the MTA and MHO groups compared with the control group following 4 weeks of treatment. These results indicated that various treatment strategies could remarkably alleviate symptoms in patients with periodontic-endodontic combined lesions. Furthermore, the data indicated that the GI and PD were not significantly different between the MTA group and MHO group $(\mathrm{P}>0.05)$. However, the GI and PD in the combined treatment group were significantly decreased compared with those in the MTA and MHO groups $(\mathrm{P}<0.05$; Fig. 3). These results suggest that the combination therapy had a superior curative effect for the patients with severe periodontic-endodontic combined lesions.

Combination treatment demonstrates superior effects to single treatments for patients with severe periodontic-endodontic combined lesions at follow-up. In order to explore the long-term efficacy of treatment strategies for patients with severe periodontic-endodontic combined lesions, the curative efficacy 2 years after treatment was statistically analyzed. The data demonstrated that the total effective rates were significantly increased 2 years after treatment in the experimental groups compared with that in the control group $(\mathrm{P}<0.05$; Table IV), indicating that the treatment strategies used in the experimental groups had a long-term effect. The data also revealed that the total effective rate in the MHO group was not significantly different to that of the MTA group ( $P>0.05$; Table IV). However, the total effective rate was significantly increased in the combined treatment group 2 years after treatment compared with that in the MTA and MHO groups, respectively $(\mathrm{P}<0.05$; Table IV). These results suggest that the long-term efficacy was not significant between MTA
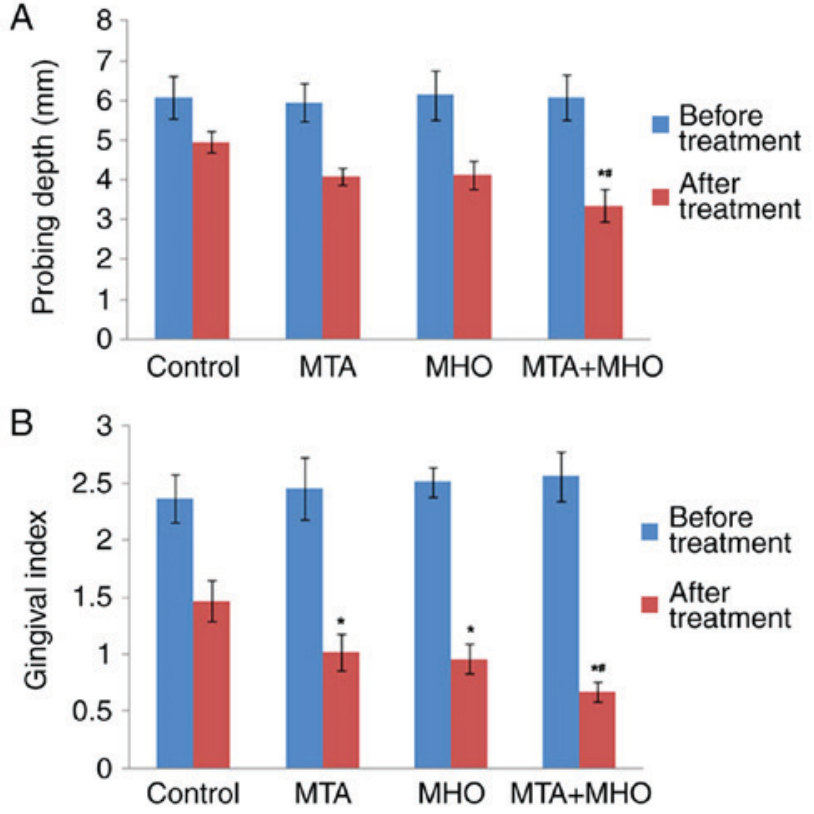

Figure 3. Comparison of probing depth and gingival index in the different treatment groups. (A) Comparison of probing depth between the different treatment groups. (B) Comparison of gingival index between the different treatment groups. ${ }^{*} \mathrm{P}<0.05$ vs. control group following treatment; ${ }^{\#} \mathrm{P}<0.05$ vs. MTA and MHO groups following treatment. MTA, mineral trioxide aggregate; $\mathrm{MHO}$, minocycline hydrochloride ointment; PD, probing depth.

treatment and MHO treatment; however, long-term efficacy was improved significantly when the combined treatment was adopted, indicating that the combination therapy has a superior curative effect.

\section{Discussion}

Periodontal pulp syndrome involves combined lesions that occur in the pulp and periodontal tissues, which is usually accompanied by severe periodontal injures (1). The clinical manifestation of periodontic-endodontic combined lesions is complicated, the course of the disease and treatment are relatively long, and its clinical prognosis is relatively poor $(19,20)$. Therefore, the clinical treatment of periodontic-endodontic combined lesions is based on the initial cause of the disease to develop the corresponding treatment plan. Dental pulp disease 
is a common disease within oral medicine and root canal filling or mummification of pulp treatment is widely used in clinics; dental adhesive, zinc oxide and phenolic resin are commonly used as root canal filling materials (21-23).

In the present study, the patients with 3-10 mm depth of periodontal pocket with severe periodontal damage were selected. Firstly, various factors affecting the treatment effect of the patients with periodontic-endodontic combined lesions were statistically analyzed using logistic regression analysis. The results demonstrated that medical history, root filling and periapical condition were the critical factors affecting the treatment of patients with periodontic-endodontic combined lesions, suggesting that these factors should be taken into account when treating this disease. Then, a combination of MHO and MTA was used for the treatment of periodontic-endodontic combined lesions, and the results revealed that the total curative efficiency of the anterior and posterior teeth was significantly increased in the combined treatment group compared with either treatment alone. This implied that combination therapy was significantly effective. In addition, the present study indicated that type I, II and III disease was significantly improved when the patients received combined treatment. All of these data suggest that the curative effect of the patients with periodontic-endodontic combined lesions was improved significantly when a combination treatment of MHO and MTA was utilized. Furthermore, GI and PD are two important indexes used to measure the comprehensive curative effect of patients with severe periodontic-endodontic combined lesions (24-26). The GI and PD of patients in the present study were measured prior to and following treatment and the results were statistically analyzed. The data demonstrated that PD and GI were decreased significantly in the combined treatment group compared with the levels in the MTA or MHO groups, the total effective rate was significantly excelled when compared with that in the control group, and the use of the drugs rarely caused local or systemic adverse reactions (data not shown). These results illustrate that following the use of MHO and MTA in combination, not only damage of the periodontal tissue was reduced, but also the tissue healed quickly, which may be related to the inhibition of collagenase activity and prevention of periodontal tissue destruction (27-31).

In conclusion, the present study utilized MTA combined with MHO for the treatment of severe periodontic-endodontic combined lesions. The data suggested that the treatment efficacy of pulp necrosis in patients was significantly improved and a therapeutic effect on the patients with apical inflammation was observed following combination treatment. Therefore, this combination treatment may be valuable in clinics for the treatment of patients with periodontic-endodontic combined lesions.

\section{Acknowledgements}

Not applicable.

\section{Funding}

The present study was supported by the Research project of Shanghai Municipal Health and Family Planning Commission (grant no. 201440401).

\section{Availability of data and materials}

The datasets generated and/or analyzed during the current study are not publicly available due to the protection of patient's privacy but are available from the corresponding author on reasonable request.

\section{Authors' contributions}

FX, ZXX and YW analyzed and interpreted the patient data regarding the hematological disease and the transplant. RHR and WS performed the histological examination of the teeth and WS was a major contributor in writing the manuscript. YQW is head of the department and helped design the study. All authors read and approved the final manuscript.

\section{Ethics approval and consent to participate}

Ethical approval was granted by the Medical Ethics Committee of Shanghai Jiaotong University (Shanghai, China; reference no. JTRT2010051531). Informed signed consent was gathered from the participants prior to initiation of the study.

\section{Consent for publication}

In the present study, the patient, parent, guardian or next of kin as appropriate provided written informed consent for the publication of any associated data and accompanying images.

\section{Competing interests}

The authors confirm that they have no competing interests.

\section{References}

1. Raheja J, Tewari S, Tewari S and Duhan J: Evaluation of efficacy of chlorhexidine intracanal medicament on the periodontal healing of concomitant endodontic-periodontal lesions without communication: An interventional study. J Periodontol 85: 1019-1026, 2014.

2. Huffaker SK, Safavi K, Spangberg LS and Kaufman B: Influence of a passive sonic irrigation system on the elimination of bacteria from root canal systems: A clinical study. J Endod 36: 1315-1318, 2010.

3. Zimet PO and Endo C: Preservation of the roots-management and prevention protocols for cracked tooth syndrome. Ann R Australas Coll Dent Surg 15: 319-324, 2000.

4. Torabinejad M, Watson TF and Pitt Ford TR: Sealing ability of a mineral trioxide aggregate when used as a root end filling material. J Endod 19: 591-595, 1993.

5. Lee SJ, Monsef M and Torabinejad M: Sealing ability of a mineral trioxide aggregate for repair of lateral root perforations. J Endod 19: 541-544, 1993.

6. Sluyk SR, Moon PC and Hartwell GR: Evaluation of setting properties and retention characteristics of mineral trioxide aggregate when used as a furcation perforation repair material. J Endod 24: 768-771, 1998.

7. Karygianni L, Proksch S, Schneider S, Vach K, Hellwig E, Steinberg T, Schulz SD, Tchorz JP and Altenburger MJ: The effects of various mixing solutions on the biocompatibility of mineral trioxide aggregate. Int Endod J 49: 561-573, 2016.

8. Prasad A, Pushpa S, Arunagiri D, Sawhny A, Misra A and Sujatha R: A comparative evaluation of the effect of various additives on selected physical properties of whitemineral trioxide aggregate. J Conserv Dent 18: 237-241, 2015. 
9. Moore A, Howley MF and O'Connell AC: Treatment of open apex teeth using two types of white mineral trioxide aggregate after initial dressing with calcium hydroxide in children. Dent Traumatol 27: 166-173, 2011.

10. Agamy HA, Bakry NS, Mounir MM and Avery DR: Comparison of mineral trioxide aggregate and formocresol as pulp-capping agents in pulpotomized primary teeth. Pediatr Dent 26: 302-309, 2004.

11. Challenger H, Lane J, Becker R, Nassiripour S and Torabinejad M: Dye leakage and modification of fast-setting mineral trioxide aggregate. J Calif Dent Assoc 43: 82-86, 2015.

12. Ravindran S and George A: Biomimetic extracellular matrix mediated somatic stem cell differentiation: Applications in dental pulptissue regeneration. Front Physiol 6: 118, 2015

13. Giovanella LB, Barletta FB, Felippe WT, Bruno KF, De Alencar AH and Estrela C: Assessment of oxygen saturation in dental pulp of permanent teeth with periodontal disease. J Endod 40: 1927-1931, 2014.

14. Xia X, Huang BX, Zhu WD and Meng HX: Effect of minocycline hydrochloride ointment on cell attachment and proliferation on titanium disks. Zhonghua Kou Qiang Yi Xue Za Zhi 47: 518-522, 2012 (In Chinese).

15. Nakao R, Takigawa S, Sugano N, Koshi R, Ito K, Watanabe H and Senpuku H: Impact of minocycline ointment for periodontal treatment of oral bacteria. Jpn J Infect Dis 64: 156-160, 2011.

16. Shigeyama M, Ohgaya T, Kawashima Y, Takeuchi H and Hino T: Modification of the physicochemical properties of minocycline hydrochloride ointment with cyclodextrines for optimum treatment of bedsore. Chem Pharm Bull (Tokyo) 48: 617-622, 2000.

17. Shigeyama M, Ohgaya T, Kawashima Y, Takeuchi H and Hino T: Mixed base of hydrophilic ointment and purified lanolin to improve the drug release rate and absorption of water of minocycline hydrochloride ointment for treatment of bedsores. Chem Pharm Bull (Tokyo) 47: 744-748, 1999.

18. Mazur B and Massler M: Influence of periodontal diseases on the dental pulp. Oral Surg Oral Med Oral Pathol 17: 592-603, 1964.

19. Rotstein I and Simon JH: Diagnosis, prognosis and decision-making in the treatment of combined periodontal-endodontic lesions. Periodontol 2000 34: 165-203, 2004.

20. Walter C, Krastl G and Weiger R: Step-wise treatment of two periodontal-endodontic lesions in a heavy smoker. Int Endod J 41: 1015-1023, 2008
21. Marques JH,Silva-Sousa YT, Rached-Júnior FJ, Mazzi-Chaves JF, Miranda CE, Da Silva SR, Steier L and Sousa-Neto MD: New methodology to evaluate bond strength of root-end filling materials. Braz Dent J 26: 288-291, 2015.

22. Lahor-Soler E, Miranda-Rius J, Brunet-Llobet L, Farré M and Pumarola J: In vitro study of the apical microleakage with resilon root canal filling using different final endodontic irrigants. J Clin Exp Dent 7: e212-e217, 2015.

23. Prestegaard H, Portenier I, Ørstavik D, Kayaoglu G, Haapasalo M and Endal U: Antibacterial activity of various root canal sealers and root-end filling materials in dentin blocks infected ex vivo with Enterococcus faecalis. Acta Odontol Scand 72: 970-976, 2014.

24. Patel SP, Kalra N, Pradeep AR, Martande SS, Naik SB, Raju AP and Singh P: Association of metabolic syndrome and periodontal disease in an Indian population. J Int Acad Periodontol 16: 98-1028, 2014.

25. AL Shayeb KN, Turner W and Gillam DG: Accuracy and reproducibility of probe forces during simulated periodontal pocket depthmeasurements. Saudi Dent J 26: 50-55, 2014.

26. Ishihata K, Wakabayashi N, Wadachi J, Akizuki T, Izumi Y, Takakuda K and Igarashi Y: Reproducibility of probing depth measurement by an experimental periodontal probe incorporating optical fiber sensor. J Periodontol 83: 222-227, 2012.

27. Geng $\mathrm{S}, \mathrm{Cao} \mathrm{C}$ and $\mathrm{Chen} \mathrm{Z}$ : The effect of non-surgical periodontal and adjunctive minocycline-HCL treatments on collagenase activity. Zhonghua Kou Qiang Yi Xue Za Zhi 35: 336-339, 2000 (In Chinese).

28. Hajishengallis G, Abe T, Maekawa T, Hajishengallis E and Lambris JD: Role of complement in host-microbe homeostasis of the periodontium. Semin Immunol 25: 65-72, 2013.

29. Anand PS, Kamath KP, Bansal A, Dwivedi S and Anil S: Comparison of periodontal destruction patterns among patients with and without the habit of smokeless tobacco use-a retrospective study. J Periodontal Res 48: 623-631, 2013.

30. Messora MR, Oliveira LF, Foureaux RC, Taba M JR, Zangerônimo MG, Furlaneto FA and Pereira LJ: Probiotic therapy reduces periodontal tissue destruction and improves the intestinal morphology in rats with ligature-induced periodontitis. J Periodontol 84: 1818-1826, 2013.

31. Konermann A, Al-Malat R, Skupin J, Keilig L, Dirk C, Karanis R, Bourauel C and Jäger A: In vivo determination of tooth mobility after fixed orthodontic appliance therapy with a novel intraoral measurement device. Clin Oral Investig 21: 1283-1289, 2017. 